\title{
Building Safer And More Inclusive Field Experiences In Support Of Planetary Science
}

A WHITE PAPER IN SUPPORT OF THE 2023-2032 PLANETARY SCIENCE AND ASTROBIOLOGY DECADAL SURVEY

\section{Corresponding Author}

Jacob Richardson (he/him)

University of Maryland College Park / NASA

Goddard Space Flight Center (GSFC)

jacob.a.richardson@nasa.gov, (301) 614-5713

\section{Co-Authors}

Nicole Whelley (she/her)

University of Maryland College Park / NASA GSFC

Patrick Whelley (he/him)

University of Maryland College Park / NASA GSFC

Moses Milazzo (he/him)

Other Orb LLC - Flagstaff, AZ

Christine Knudson (she/her)

University of Maryland College Park / NASA GSFC

Rodrigo Romo (he/him)

Pacific International Space Center for Exploration

Systems (PISCES), Hilo HI

Shannon Kobs Nawotniak (she/her) Idaho State University

\section{Hyperlink to 51 Co-signatories and Long-form Citations of this White Paper}

\section{https://bit.ly/3hxdoat}

\section{Abstract}

Field expeditions in support of planetary science are important to advance our understanding of planetary processes and enhance the science community through training and close, often interdisciplinary collaborative efforts. Still, field work faces unique safety risks and barriers to entry, due to the physical nature of the field but also from team behavior and sometimes inhospitable communities near common field sites. Field teams need to be resilient to fieldsite hazards and self-supportive to improve safety and accessibility. We call on NASA, the NSF, and the planetary science community to foster resilient field teams by 1) requiring field safety plans from funded field teams; 2) providing both physical and mental safety field training; 3) developing a NASANNSF-wide, field-specific code of conduct; 4) supporting field experiences for students and early career researchers; 5) engaging with scientists and communities local to field sites of interest; 6) holding NASA and NSF funded field teams accountable for providing safe workplace environments in the field; and finally 7) thinking critically about institutional safety requirements that are designed for traditional workplaces and not the field. 


\section{The Importance of Field Expeditions in Planetary Science}

The in situ study of landforms and locations on Earth that are analogous to environments and geologic units found on other planets is a fundamental tool within planetary science (Whelley et al., 2020; Hodges and Schmitt, 2019). Virtually all scientific interpretations of the evolution of planetary surfaces and climates are rooted in our understanding of how these processes occur or occurred on Earth. Exploring Earth analog environments through field science advances planetary science in two ways. First, field science trains scientists to better observe and interpret features on Earth (Levine et al., 2007; Elkins and Elkins, 2018; Mogk and Goodwin, 2012). Second, field science enables the in situ exploration of planetary features and environments without the complexities inherent to a space mission, as Earth is itself a planet (Garry and Bleacher, 2011; Whelley et al., 2020).

The state of field work, despite the myriad science and workforce benefits, is far from perfect. The planetary science community can address persistent issues and make field work a safer, more inclusive environment that incubates new avenues of investigation between our planet and our neighbors. In response to the goal of the Planetary Science and Astrobiology Decadal Survey to provide exposition of the state of the field, including "the creation of safe workspaces", we submit the following recommendations to NASA, the NSF, and the planetary science community at large to promote positive change in field science: 1. For all NASA and NSF-funded field work, require a field safety plan that outlines physical and mental health safety, strategies for reducing barriers to field work, and coordination with local communities. 2. Provide both physical and mental health safety training to all funded projects that include field work. 3. Develop a Code of Conduct for all NASA and NSF funded field work. 4. Support field training of students and early career scientists. 5. Encourage field teams to include local scientists and engage communities. 6. Provide avenues to appeal institutional requirements that make for unsafe practices in the field. 7. Hold NASA and NSF funded teams accountable for field safety. Below we detail issues related to field safety, etiquette, and barriers to the field that provide context to these recommendations.

\section{Physical and Mental Safety in the Field Physical Safety}

Hazards - threats to safety that can be natural (e.g., thunderstorms) or anthropogenic (e.g., foodborne illness) - are present in every workplace, and organizations work to identify and mitigate exposure to them. In field expeditions, hazards are different from traditional workplace settings and effective field teams systematically work to identify the hazards present at their field sites. Once hazards are identified, field teams should develop safety plans to limit exposure to hazards. Two ways to limit exposure are hazard avoidance and protection. Hazard avoidance can range from staying away from cliff faces to checking the weather and maintaining field vehicles. Protection from exposure to hazards can include using personal protection equipment (PPE), following the Buddy System, and maintaining first aid kits. Additionally, establishing "Stop Work Authority" for all participants in field work can limit unsafe practices if field teams empower all participants to act when safety concerns arise. Hazard avoidance and protection can also be addressed during field work preparation by providing appropriate safety training based on the field setting. Some effective training courses include First Aid and CPR courses provided by the Red Cross or 
National Outdoor Leadership School (NOLS). For more complex or larger field campaigns, emergency response training including the Federal Emergency Management Agency's Incident Command System can be useful, especially for field team leadership.

\section{Mental Safety}

Hazards in the field are not limited to rock falls and flat tires. In addition to hazards related to physical health in field settings, unique hazards to mental health (including social and behavioral health) are also present in the field. Of primary concern, rates of harassment in field settings are egregiously high with a majority (64\%) of participants in field research reporting personal experience of sexual harassment in a recent survey (Clancy et al., 2014). Behavioral hazards are of special concern in the field because of the intimate nature of field campaigns. Groups are often in unpopulated or unfamiliar areas, which limits contact with the outside world and participants' existing social networks. Field work is generally a 24-hour a day commitment for the duration of the expedition and cohabitation in campgrounds or hotels limits the ability to create distance between participants.

These characteristics of field work make it an intimate setting where people can work closely with each other to achieve common goals but also where tensions can escalate if left unchecked. In the field, no participant is immune to engaging in problematic behavior that can adversely affect team morale. Effective field teams can limit this, similar to limiting exposure to physical hazards, through safety planning and preparation. It is imperative that field teams recognize that all participants might engage in problematic behavior, that problematic behavior is compounded by the power structure in the field, and that despite this, all participants can be empowered to promote prosocial behavior in the field. Training, such as Bystander Intervention Training ${ }^{1}$, provides skill sets to participants to identify and address problematic behavior as it occurs.

\section{Mental health and physical health are not mutually exclusive topics and should not be treated as such. To students and professional researchers who are members of} marginalized communities, issues with harassment in the field that might be considered to be mental health issues may be experienced as risks to physical health. For example, problematic behavior that might go unrecognized to other researchers can be understood as "dog-whistle" language or microaggressions. When this behavior is left unchecked in an environment with little chance to create distance between participants, it has the potential to develop into physical confrontations and even violence in the field. For example, regarding gender harassment, Barthelemy et al. (2016) found that even in traditional workspace environments "without immediate investigations and appropriate responses, unchecked microaggressions will give the signal that it is acceptable for males to be perpetrators, and women have little to no recourse to appropriate intervention." LGBTQ+ scientists in physics in particular have been found to experience isolation related to their sexual orientation or gender identity, which is in part due to a culture that expects closeted behavior (Atherton et al., 2016, Vander Kaaden et al., 2020). This feeling of isolation can be exacerbated in field settings due to its remote and unfamiliar environment. Situations

\footnotetext{
${ }^{1}$ e.g., the USGS StepUp! Employee Empowerment Strategies, https://www.usgs.gov/about/organization/science-support/human-capital/bystander-intervention-usgs-seesworkshop
} 
where microaggressive and/or homophobic behavior are permitted but expectations of closetted behavior are present create an unsafe imbalance of power in the field.

Compounding problems of harassment along single discriminatory axes, studies repeatedly find that scientists belonging to multiple historically excluded communities (e.g., women of color) experience more harassing behavior than scientists belonging to single marginalized communities (Clancy et al., 2017; Marín-Spiotta et al., 2020). More than the sum of their parts, sexual and racial harassment in combination have interactive effects on mental health (Marín-Spiotta et al., 2020). Battling problematic and harassing behavior in the field requires buy-in from the entire field team, especially leadership. It is easiest to respond to problematic behavior immediately instead of avoiding it, sometimes through "microinterventions" (Sue et al., 2019), or better yet through preventative training, communication, and example-based leadership from field leaders.

Recommendation \#1: Require a Field Safety Plan that outlines physical and mental safety, strategies for reducing barriers to field work, and coordination with local communities for all NASA and NSF funded field work.

We envision such plans to be included with research proposals to NASA and the NSF. Similar to Data Management Plans required for most NASA research proposals ${ }^{2}$, Field Safety Plans should be external to the page-limited Science and Technical Management Plans. In an effort to reduce writing burden for field groups, NASA should provide a Safety Plan template. We provide a potential template at the end of this document.

Recommendation \#2: Provide physical and mental safety training to funded projects that include field work.

NASA should fund or provide free safety training, with equal weight given to physical and mental safety, to planetary science field teams. This can be done by allocating funding for training to projects that include field work, or by providing this training virtually and at community science meetings, such as DPS, LPSC, and AGU.

\section{Behavior in the Field}

Inequities in field experiences might be one of the reasons that geoscience remains the least diverse discipline within science and engineering fields (Gannet Hallar et al., 2018; Kortz et al., 2018). These inequities lead to harassment that is all too common: The SAFE (Survey of Academic Field Experiences) Report by Clancy et al. (2014) found 64\% of respondents had personally experienced sexual harassment (70\% of women and $40 \%$ of men). Additionally $20 \%$ of respondents reported sexual assault in the field (26\% of women, $6 \%$ of men). These experiences are not only detrimental to the field as a productive workplace (Clancy et al., 2014; Popovich and Warren, 2010), these experiences are violence, and as such, should never be tolerated.

Codes of Conduct for field work outline community norms, expectations, and responsibilities for the behavior and actions of the field team. Fewer than $40 \%$ of respondents in the SAFE Report (Clancy et al., 2014) recalled seeing a Code of Conduct during field work, and avenues to report harassment or assault is often less than transparent (Nelson et al., 2017). Ensuring that behavioral expectations of the entire field

\footnotetext{
${ }^{2}$ NASA Data Management Plan FAQ, https://science.nasa.gov/researchers/sara/faqs/dmp-faq-roses
} 
team are clear, understood, and accepted by all field participants is vital to both the physical and mental safety of field scientists. While NASA-funded field work can be done by scientists at multiple different institutions, to non-academics, all NASA funded groups are seen as "NASA" while doing fieldwork, regardless of their institution. It is important that funded research groups understand their implicit role as ambassadors for NASA and act accordingly. Therefore, it is recommended that NASA create a Code of Conduct specifically for all NASA funded work to ensure that there are inclusive, safe, and inspiring social norms, expectations, and behavior from all NASA funded field work as well as a clear structure of accountability (Diniega et al., 2020).

\section{Recommendation \#3: Develop a Field Work Code of Conduct specific for all NASA and NSF funded field work. \\ NASA and the NSF can be leaders in physical and mental safety in field work, but in order to do so we need to have physical and mental safety policies that have clear definitions and reporting procedures (Diniega et al., 2016; Diniega et al., 2020).}

\section{Barriers to accessing the Field}

A key benefit to field work is how it promotes community within the planetary and geosciences disciplines (Streule and Craig, 2018). In the geosciences, students first self-identify as geoscientists during initial field experiences (Kortz et al., 2019; Atchison et al., 2019), which can help retain students in this related science discipline (Levine et al., 2007; Gannet Hallar et al., 2018). Physical, outdoor experiences are known to link classroom and theoretical concepts to real world examples, which encourages interest in further research (Kortz et al., 2019). However, In a culture that values field work but does not work to make it inclusive or safe, field work becomes a practice that provides benefits to some and detriments to others, negating its role in the geoscience career pipeline.

Despite the benefits to the planetary science community and its researchers, barriers large and small are present that discourage or outright inhibit researchers from participating in field work. Often these barriers are highest for people from backgrounds already historically excluded from the sciences in general. These barriers between historically excluded researchers and the field serve to further filter geoscientists into a homogeneous workforce lacking diversity.

Field campaigns, including critical and sometimes mandatory training experiences (Sima, 2020), are often conducted without thought to accessibility for students or researchers with physical, sensory, or developmental disabilities. Atchison et al. (2019) identifies this lack of accessibility to the field as a critical issue in excluding students with disabilities from developing a self-identity as a scientist, which is an important element in career retention (Kortz et al., 2019). In addition to field sites being inaccessible due to rough terrain or backcountry living conditions, travel to remote locations often require additional logistical preparation and cost for people with disabilities that often exceed reimbursement limits set by institutions (Piatek et al., 2020). The NSF's GEOPaths Program ${ }^{3}$ does potentially fund research that can enable more accessible field work. We encourage NASA to take a similar role in funding pathways to train the next generation of planetary scientists in the field.

${ }^{3}$ https://www.nsf.gov/funding/pgm summ.jsp?pims id=505169 
Field groups often ignore issues of gender and sex in preparation and safety planning. As previously mentioned, the prevalence of sexual violence and generally unwelcoming environments (Clancy et al., 2014) can dissuade people from sexual and gender minority groups from participating in field work. Even in groups that have thorough and effective anti-harassment policies, more preparation and consideration to issues that are often gendered can provide a welcoming environment that encourages scientists to join field work. These practices include normalizing essential practices like going to the bathroom and menstruation hygiene in the field (Greene et al., 2020), and providing plans for caregivers (e.g., planning for childcare and eldercare back home, lactation needs in the field). Properly inclusive bathroom policies serve any potential team member, including trans participants and participants who administer medication in private.

The geosciences are also the least racially diverse field within STEM fields (Huntoon and Lane, 2007; Kortz et al., 2019, Gannet Hallar et al., 2018). This might be attributed to inequity in access to field experiences stemming from a number of reasons. First, while field experiences are important for retention in the geosciences (Kortz et al., 2019; Gannet Hallar et al., 2018), the "great outdoors" in the United States have historically (and erroneously) been framed as a "white space" (Finney, 2014). Second, students from communities who have been historically excluded from financial prosperity based on their race in the United States might be less often able to pay up front for travel and lodging to the field, often a requirement for field participation (Gannet Hallar et al., 2018).

Real and perceived threats to personal safety due to communities near field sites in the United States and abroad impact several marginalized communities of geoscientists who might justifiably avoid field work. Many white supremacist and homophobic hate groups in the US have roots in the american southwest ${ }^{4}$, which hosts many world-renowned planetary analog field sites. Additionally one-third of LGBTQ+ geoscientist respondents to a recent survey (Olcott and Downen, 2020) reported refusing field work altogether due to concerns about personal safety. Note that "perceived" threats to personal safety are real mental health and safety concerns even if physical safety is never explicitly threatened.

In part, careful planning and effective training can reduce or remove some of these barriers to help include new scientists in field activities. Because these barriers often impact people at the beginning of their education or training in geosciences, we recommend that specific emphasis is placed on continued support of training experiences that are genuinely inclusive to all early career researchers that are interested in field activities.

\section{Recommendation \#4: Support training of students and early career scientists performing field work.}

Fund undergraduate to postdoctoral-level field experiences to relevant planetary analog sites $^{5}$. Include travel stipends for trainees to lower economic barriers. Support making field work accessible for trainees with disabilities and inclusive (logistically and geographically) for the widest range of trainees. Don't require trainees to be part of funded NASA projects and instead actively recruit trainees from communities historically excluded in STEM.

\footnotetext{
${ }^{4}$ Southern Poverty Law Center, Hate Groups in the United States https://www.splcenter.org/hate-map

${ }^{5}$ e.g. the Planetary Volcanology Workshop, https://www.higp. hawaii.edu/ harold/volcano wkshop/
} 


\section{Valuing communities at field sites}

Field work in rural areas of the United States and international field work often takes place near communities that have less access to educational opportunities that can lead to careers in planetary science. Engaging with local communities and including students and scientists with connections to field sites of interest can therefore expand the planetary science community. Genuine connections with local experts also can enable field teams to better plan field work that is more respectful of community values and more aware of property rights and safety concerns. For example, all non-Tribal members who hike or do field work on Diné Bikeyah (The Navajo Nation) are required by the Nation's laws to apply for and receive a permit prior to beginning those activities.

Many analog field sites have a strong cultural significance to indigenous people and/or cultures. It is critical for field teams to do research ahead of time regarding the indigenous people and the cultural significance that the site in question plays in their heritage. As scientists looking to advance our knowledge in exploring other planets, it is our responsibility to have the utmost respect for local culture in order to avoid any potential offense to indigenous groups. This is especially important following a decade where U.S. geology and astronomy continued to set particularly bad examples of disregard for communities' culture and heritage with projects like the Dakota Pipeline and the Thirty Meter Telescope (Kahanamoku et al., 2020).

Recommendation \#5: Encourage research teams participating in field work to include researchers and students with connections to their field sites within their science team and engage with local communities before, during and after field work.

Where proposed field work takes place on tribal or indigenous lands within the U.S. and abroad, NASA and the NSF should require proof of permits or plans to acquire permits prior to funding the work. NASA and the NSF should encourage field teams to genuinely engage with local cultural practitioners or elders if the analog site is of cultural importance to indigenous communities and engage the public local to all field sites.

\section{Accountability}

Codes of conduct, safety plans, and training can lead to safer field work for all. However, it is of vital importance to not only ensure field teams have access to these best practices, but to hold teams accountable to enacting, enforcing, and ensuring safe cultures in the field (St. John et al., 2016). NASA can lead in this effort by expanding and publicizing reporting requirements from institutions that receive funding from NASA.

Recommendation \#6: Hold NASA and NSF funded teams accountable for safety. We recommend that in-place anti-harassment reporting requirements ${ }^{6}$ are broadened to include violations for creating unsafe working conditions, specifically in field settings.

NASA can also help promote safe field practices by working with field teams to ensure that safety procedures at NASA facilities are also applicable to field environments. As a primary example, the requirement that Civil Servants cannot ride in vehicles driven by contractors ${ }^{7}$

${ }^{6}$ NASA Grant and Cooperative Agreement Manual (GCAM), https://prod.nais.nasa.gov/pub/pub library/srba/documents/Grant and CooperativeAgreementManual.pdf

${ }^{7}$ NASA General Travel Requirements, Section 5.8,

https://nodis3.gsfc.nasa.gov/displayDir.cfm?Internal ID=N PR 97100001 \&page name=Chapter5 
might be appropriate for traditional workplace settings but is not a safe field practice where long or technical drives in remote locations necessitate alternate drivers.

Recommendation \#7: Provide avenues for appeals of institutional requirements at NASA that make for unsafe practices in the field.

To support safe field practices, create avenues for appeals of blanket requirements and allow for safe alternatives to be used instead.

\section{Safety Plan Template}

Here we provide sample questions that can enable panel reviewers to evaluate the safety plans of proposing field research teams. We encourage questions that are broad enough to guide proposers in planning safe campaigns whether field work is local or remote.

\section{What are the field-setting specific hazards at the proposed field site?}

What environmental, personal, and equipment hazards are present?

2. What is the field team's plan to reduce exposure to field hazards?

What protection equipment will the team use? What is the team's ability to communicate with each other and with the outside world at the field site? Does the proposed schedule have flexibility to allow for avoidance of temporary field hazards?

3. What is the field team leadership structure?

Who will be the field lead? Is there a need for a logistics specialist and, if so, who will this be? Is there a need for a safety officer and, if so, who will this be? Who are the field-site local points of contact within the leadership structure?

4. What is the communication plan for safety information?

Will there be a Code of Conduct available to all participants? What is the contact information for local emergency services? How will issues and emergencies that do not require emergency services be reported?

5. What safety training will take place before going into the field?

What physical, mental, and management training will take place or has taken place?

Who on the team has and/or will take this training?

\section{Short Form Citations}

Full Citations available at https://bit.ly/3hxdoat

Atchison, C. L., et al. (2019) J. Geosci. Edu.; Atherton, T. J., et al. (2016), Am. Phys. Soc.; Barthelemy, R. S., et al. (2015) PERC; Clancy, K. B. H., et al. (2014) PLOS ONE; Clancy, K. B. H., et al. (2017) JGR Planets; Diniega, S., et al. (2016) Eos; Diniega, S., et al. (2020) NAS Decadal White Paper; Elkins, J. T. \& Elkins, N. M. L. (2007). J. Geosci. Edu.; Finney, C. (2014) UNC Press; Gannet Hallar, A., et al. (2010) J. Geosci. Edu.; Garry, W. B., \& Bleacher, J. E. (2011) GSA S.P. \#483; Greene, S., et al. (2020); Hodges, K. V. \& Schmitt, H. H. (2019) Sci. Advances; Huntoon, J. E., \& Lane, M. J. (2007) J. Geosci. Edu.; Kahanamoku, S., et al. (2020); Kortz, K. M., et al. (2020) J. Geosci. Edu.; Levine, R., et al. (2007). J. Geosci. Edu.; Marín-Spiotta, E. et al. (2020) Adv. Geosci.; Mogk, D. W., \& Goodwin, C. (2012) GSA S.P. \#486; Nelson, R. G., et al. (2017) Am. Anthropol.; Paitek, J. L. et al. (2020) NAS Decadal White Paper; Richey, C. R., et al. (2019) BAAS; Sima, R. (2020) Eos; St. John, K., et al. (2016) J. Geosci. Edu.; Streule, M. J., \& Craig, L. E. (2016) J. Geosci. Edu.; Sue, D. W., et al. (2019) Am. Psycol.; Vander Kaaden, K. E., et al. (2020). NAS Decadal White Paper; Whelley, P. L., et al. (2020) NAS Decadal White Paper. 